\title{
Representación, mandato y poder. Una encrucijada entre el derecho de forma y de fondo
}

\section{POR MAURO F. LETURIA $(*)$, SOLANGE C. NUGOLI $(* *)$ Y ADRIÁN E. GOCHICOA $(* * *)$}

\begin{abstract}
Sumario: I. Introducción.- II. Representación.- III. Representación voluntaria: poder y ratificación.- IV. Mandato.- V. Forma del poder, del mandato y la regulación procesal.- VI. Jurisprudencia.- VII. El Anteproyecto de Código Procesal Civil, Comercial y de Familia de la provincia de Buenos Aires.- VIII. Bases para la Reforma Procesal Civil y Comercial para el "Anteproyecto de Nuevo Código Procesal Civil y Comercial de la Nación".- IX. Conclusión.- X. Bibliografía.
\end{abstract}

Resumen: el análisis realizado en el presente importa un profundo estudio y delimitación conceptual del "mandato", la "representación" y el "poder" a la luz del Código Civil y Comercial de la Nación. Asimismo, se trabajarán sus repercusiones jurisprudenciales, cimentadas sobre los Códigos de forma de la Nación y de la provincia de Buenos Aires. El eje central del trabajo se apuntala en la forma como

(*) Procurador, abogado y escribano. Esp. en Docencia Universitaria. Doctorando de la Facultad de Ciencias Jurídicas y Sociales, Universidad Nacional de La Plata, UNLP. Realizó estudios de postgrado sobre Derechos de Autor, Universidad Complutense de Madrid. Prof. Derecho Civil III, Introducción al Pensamiento Científico y Derecho Marítimo y Aeronáutico y Prof. de Posgrado de la Esp. en Documentación y Registración Inmobiliaria, Facultad de Ciencias Jurídicas y Sociales, Universidad Nacional de La Plata, UNLP. Prof. Titular Prácticas Profesionales II y Derecho de la Navegación, Facultad de Derecho y Ciencias Políticas, Universidad Católica de La Plata, UCALP. Prof. Derecho Penal I parte general y Derecho Marítimo y Aeronáutico, Facultad de Derecho, Universidad del Este. Prof. de Metodología de la Investigación, Facultad de Diseño y Comunicación, Universidad del Este. Docente invitado en la Universidad Complutense de Madrid. Prof. de posgrado de la Universidad Austral. Oficial de la Justicia Federal Argentina. Becario Doctoral del Ministerio de Educación de la Nación. Secretario de Extensión y de Investigación, Facultad de Derecho, Universidad del Este.

(**) Abogada. Esp. en Derecho Ambiental, Aeronáutico y Espacial. Adscripta Cátedra II Derecho de la Navegación (Marítimo, Fluvial y Aéreo), Facultad de Ciencias Jurídicas y Sociales, Universidad Nacional de La Plata, UNLP. Coord. de Clínicas Jurídicas de Derecho de los Consumidores y Usuarios, Facultad de Ciencias Jurídicas y Sociales, Universidad Nacional de La Plata, UNLP. Jefa de Trabajos Prácticos, Universidad de Católica de La Plata, UCALP. Asesora en la Dirección General de Cultura y Educación de Buenos Aires.

$\left.{ }^{(* * *}\right)$ Abogado. Auxiliar Docente Contratos Modernos, Tecnicatura de Martillero y Corredor, Facultad de Ciencias Jurídicas y Sociales, Universidad Nacional de La Plata, Universidad Nacional de La Plata, UNLP. 
elemento esencial de la "representación procesal voluntaria", focalizándose en la regulación que se realizaba en el Código de Vélez, los cambios a la luz del nuevo Código Civil y Comercial, y la regulación que realiza el Código Procesal Civil y Comercial de la provincia de Buenos Aires y el de Nación.

Palabras claves: representación - mandato - poder

\section{Representation, mandate and power. A crossroads between the law applica- tion and interpretation.}

Abstract: the analysis carried out in the present requires an in-depth study and conceptual delimitation of the "mandate", the "representation" and the "power" by the Civil and Commercial Code of the Nation. Furthermore, its jurisprudential repercussions will be worked on, based on the Codes of the Nation and the Province of Buenos Aires. The central axis of the work is based on the form as an essential element of the "voluntary procedural representation," focusing on the regulation made in the Velez's Code, the changes by the new Civil and Commercial Code, and the regulation that performs the Civil and Commercial Procedure Code of the Province of Buenos Aires and the Nation.

Keywords: representation - mandate - power

\section{Introducción}

En primer lugar, vale aclarar que, en las respectivas jurisdicciones el Código de Vélez fue anterior a la sanción de sus respectivos códigos procesales, por lo cual resulta lógico que estos se adaptasen a dicha normativa. Esta metodología puede observarse, en lo que a la representación procesal se refiere.

De este modo, en los códigos de forma de la provincia de Buenos Aires y de Nación, donde en el Título 2 referido a las Partes (Del proceso) en el capítulo 2 referido a la Representación procesal, se señala la necesidad de acreditar la representación que se invoca cuando se intervenga en el proceso por un derecho que no es propio o es de otro. En ambos casos, en el artículo 46 se alude a "acompañar los documentos que acrediten el carácter que enviste", y en el artículo 47 se señala que

(...) Los procuradores o apoderados acreditarán su personalidad desde la primera gestión que hagan en nombre de sus poderdantes, con la pertinente escritura de poder. Sin embargo, cuando se invoque un poder general o especial para varios actos, se lo acreditará con la agregación de una copia íntegra firmada por el letrado patrocinante o por el apoderado. De oficio o a petición de parte, podrá intimarse la presentación del testimonio original (...). 
Por cuanto el Código de Vélez no desarrolló una teoría general de la Representación (a diferencia del actual Código Civil y Comercial) la doctrina la elaboró a partir de la regulación que aquel ordenamiento realizaba respecto del contrato del mandato.

En consecuencia, ante la exigencia del artículo 1184 inciso 7 del Código de Vélez que imponía que "(...) Deben ser hechos en escritura pública, con excepción de los que fuesen celebrados en subasta pública: (...) $7^{\circ}$ los poderes generales o especiales que deban presentarse en juicio (...)". La doctrina procesalista entendió que los artículos 46 y 47, señalados, referían que la acreditación era mediante la presentación de la correspondiente escritura pública y no con una copia simple de la misma, quedando este caso a salvo únicamente para la excepción contemplada, es decir, para cuando se invocara un poder general o especial para varios actos (caso en el que se podía acreditar con la agregación de una copia íntegra firmada por el letrado patrocinante o por el apoderado).

Arribado el nuevo Código Civil y Comercial (CCiv. y Com.), se modificó la exigencia de la escritura pública como forma de instrumentar un poder general o especial para presentar en juicio, sin que con ello se adaptasen la letra de los ordenamientos procesales, a lo que se suma en la provincia de Buenos Aires la experiencia del expediente electrónico.

Por las razones expuestas, resulta vital realizar el esfuerzo para delimitar la conceptualización de la representación, el mandato y el poder, a fin de evitar confusiones en cuanto a la aplicación de la normativa específica.

\section{Representación}

A continuación se analizan diferentes conceptualizaciones sobre la representación, ancladas en diversas tesituras sobre su descripción.

En tal sentido Rivera y Crovi señalan que existe representación cuando:

(...) una persona - representante- declara su voluntad en nombre y por cuenta de otra persona - representado- en virtud de una facultad apropiada o suficiente, de tal modo que los efectos se producen directa e inmediatamente en el representado, como si el mismo hubiera ejecutado el acto (2016, p. 614).

Asimismo, Mosset Iturraspe enseña que nos encontramos frente a una representación: 
(...) cuando una persona - representante- declara su voluntad en nombre y por cuenta de otra persona - representado-. Son dos en consecuencia, los presupuestos de la representación directa: declarar en nombre ajeno quiere decir hacer saber al tercero que quien contrata -representante- no es aquel que adquiere los derechos o contrae las obligaciones por ser parte en la relación jurídica - el representado-, y actuar por cuenta ajena o en interés ajeno significa actúa para satisfacer fines o intereses de otro (1998, p. 210).

Por su parte Alterini afirma que hay representación “(...) cuando un sujeto realiza un acto jurídico en interés de otro, de manera que la actuación de aquél (representante) compromete directamente a éste (representado)" (Alterini, 1999, p. 309).

El Dr. Lorenzetti lo ha definido como “(...) una técnica jurídica que permite imputar efectos directos a una persona por la actuación de otra, sea que exista un acto voluntario o bien un comportamiento típico objetivado que autorice a hacerlo" (Lorenzetti, 2003, p. 415).

Borda define a la representación señalando que “(...) es la actuación de una persona en interés de otra, a la cual sustituye en uno o más actos jurídicos, y se la admite o es impuesta, según el caso, por razones de interés General (...)" (Borda, 2016, p. 598).

Rivero y Medina al comentar el CCiv. y Com. la conceptualizan como:

(...) un amplio género jurídico que permite a una persona otorgar en nombre y por cuenta de otro, actos jurídicos entre vivos, con la salvedad de aquellos que únicamente puedan ser otorgados por el titular del derecho, por ser personalísimos del mismo o intuitu personae (2015).

Lorenzetti analizando el CCiv. y Com. de la Nación la describe como:

(...) un instituto autónomo, una situación jurídica que se presenta como medio eficaz para celebrar actos jurídicos entre vivos ante la imposibilidad de hacerlo personalmente, salvo casos excepcionales donde esta situación es insalvable por exigencia expresa de la ley, y la persona debe actuar exclusivamente por sí —explicándose que- esta pretendida autonomía de la representación se desprende de la misma definición del instituto a la que se ha arribado tras un largo período de debate. En este estadio de evolución del Derecho, aceptamos que 'la representación' más que un negocio jurídico es una 'situación jurídica' que emerge o nace de diversas fuentes (2015, p. 419). 
En su obra López de Zavalía analiza lo que conceptualiza como representación directa, representación indirecta, ambas en su faz activa y pasiva, tomando como eje central las nociones de "acción de representar" y "efecto de representar". Así señala que en relación con la primera (acción) "la nota necesaria y suficiente para que ella se dé, es que alguien observe una conducta cuyo significado sea el de estar formulando una declaración en nombre de otro", y en relación con la segunda (efecto) "consiste en que lo declarado por el representante vale como si lo hubiera sido por el representado" (López de Zavalía, 1997, p. 489). Aclara que para producirse este efecto representativo debe existir una particular forma de legitimación, ya sea antes o después del acto o bien depender de la suerte de este. Señala que existe representación directa cuando se dan estas dos notas, es decir, cuando se actúa en nombre de otro y los efectos de esa actuación caen en forma directa en ese otro. Mientras que hay representación indirecta cuando se actúa en nombre propio y los efectos jurídicos de lo actuado solo en manera indirecta pasan a otra persona, solo con una ratificación posterior. Concluyendo que la representación indirecta refiere simplemente a un fenómeno, no unido estrechamente a la representatividad y cuyos efectos se podrían explicar con los principios de la acción subrogatoria.

Del breve compendio de definiciones puede apreciarse una conceptualización amplia que repara solo en los efectos o según el interés en que se actúe, y una más restrictiva si solo se repara en el hecho de en nombre de quien se actúa. Así, en el primer sentido se conceptualiza a la representación como la actuación en nombre o no de otro siempre que dicha actuación sea en interés ajeno, produciendo efectos jurídicos inmediatos o mediatos en la esfera jurídica de aquel en cuyo interés se actúa de acuerdo con que se actué con legitimación previa (poder-autorización) o posterior (ratificación). Por otro lado, se lo conceptualiza restrictivamente si solo se entiende a la representación como la actuación en nombre e interés de otro contando con la legitimación previa (poder-autorización).

Teniendo en cuenta los lineamientos que realizó la doctrina nacional en torno a la conceptualización de la "teoría de la representación", puede entenderse la representación como un:

(...) fenómeno jurídico en cuya virtud una persona gestiona asuntos ajenos, actuando en nombre propio o en el del representado, pero siempre en interés de éste, autorizando para ello por el interesado o en su caso por la ley, de forma que los efectos jurídicos de dicha actuación se produzcan directamente o indirectamente en la esfera jurídica del representado (...) (Leturia, 2016, p. 300).

En consecuencia, en la representación se trasladarán los efectos jurídicos de lo actuado por el representante (acto u hecho jurídico) a la esfera jurídica del 
representado, subyaciendo en los efectos jurídicos que se imputan o trasladan una vinculación con un tercero, de tal manera que la relación o situación jurídica se produce entre el representado y este tercero. Puede advertirse que dentro de este fenómeno jurídico existe, en los hechos, por un lado, una relación entre el representante y el representado, una relación entre el representante y el tercero, y por último una tercera relación entre este tercero y el representado. Jurídicamente, la teoría de la representación permite que la relación jurídica principal se establezca entre el tercero (que se relaciona con el representante) y el representado, lo que no quita que existan efectos jurídicos en las tres relaciones que se suceden.

En este orden de ideas, Mosset Iturraspe (1998) señala que la teoría de la representación se refiere exclusivamente al vínculo entre el representante y el tercero, siendo ajenas la causa de la representación como las relaciones internas que se dan en ella. Explicando que la voluntad y el interés jurídico se encuentran disociados, ya que la voluntad material la expresa el representante mientras que el interés jurídico pertenece al representado.

El CCiv. y Com. en el artículo 358 limita la teoría de la representación a la realización de actos entre vivos, salvo para aquellos actos jurídicos en los que la ley exige sean realizados solo por el titular del derecho. Asimismo, distingue las clases y fuentes de representación en voluntaria "cuando resulta de un acto jurídico", legal "cuando resulta de una regla de derecho" y orgánica "cuando resulta del estatuto de una persona jurídica". Por su parte, Lorenzetti (2015) señala que en estas clases de representación subyacen tres finalidades diferentes, así en la voluntaria la finalidad es la conveniencia personal del representado, en la legal la necesidad de protección del representado a través de su tutela, y en la orgánica dar solución a la limitación fáctica que tienen las personas jurídicas.

En cuanto a los efectos el artículo 359 señala que

(...) los actos celebrados por el representante en nombre del representado y en los límites de las facultades conferidas por la ley o por el acto de apoderamiento, producen efecto directamente para el representado $(\ldots)$.

Esta idea se adecua a la representación directa, ya que la traslación de los efectos jurídicos se produce directamente entre el tercero que celebra el acto con el representante y el representado, pero en el caso de la denominada representación indirecta esa traslación al producirse indirectamente, los efectos jurídicos se imputan o se producen entre los que celebran el acto (tercero y representado) y luego mediante otro acto esos efectos se trasladan de la esfera jurídica del representante a la esfera jurídica del representado. 
https://doi.org/10.24215/25916386e003

Por otro lado, para quienes toman a la representación en su concepción amplia, los efectos están descriptos también en el artículo 369 donde se regula la ratificación. Esta suple el defecto de representación, convirtiendo a la actuación por autorizada con efecto retroactivo al día del acto. Sin perjuicio de ello, será inoponible a terceros que hayan adquirido derechos con anterioridad.

\section{Representación voluntaria: poder y ratificación}

En la actualidad, la doctrina es unánime en la distinción entre representación, poder y mandato en el ámbito de la representación voluntaria. Sin embargo, existe una conexión entre los conceptos de poder y representación.

En referencia a los efectos de la representación y a su extensión, los artículos 359 y 360 del CCiv. y Com., señalan como límite las facultades conferidas por la ley o por el acto de apoderamiento, lo que implica que esta particular legitimación encuentra sus límites en la causa o la fuente que le da origen. En los supuestos de representación necesaria (legal y estatutaria u orgánica), se desarrolla la teoría del oficio o cargo privado (Betti, 2000) o se habla de la autorización para justificar jurídicamente esta legitimación, mientras que en la representación voluntaria se utiliza la figura del poder.

En este orden de ideas refiriéndonos al poder, la doctrina lo identifica con "el acto de apoderamiento" o bien con "la forma de instrumentar la facultad o facultades que se concede en la representación" (Leturia, 2016, p. 304).

De este modo, la idea de poder se vincula con una:

(...) situación jurídica preexistente o posterior al acto o hecho jurídico realizado en interés ajeno por la cual se puede imponer un orden vinculante a los intereses jurídicos nacidos de ese acto o hecho jurídico, imputando directamente los mismo a la esfera jurídica de aquel en cuyo interés se realizó (Leturia, 2016, p. 304).

Así, mientras la representación como fenómeno jurídico abarca la acción y efecto de representar, refiriéndose a la relación entre el representado y el tercero con quien realiza el acto o hecho jurídico (relación externa) y a la imputación (directa o indirecta) de los efectos jurídicos de ese acto o hecho a la esfera jurídica de aquel en cuyo interés se actúa, la idea de "poder" (poder y ratificación, para el caso de representación voluntaria) se refiere al porqué del encauzamiento de los efectos jurídicos de ese acto o hecho, produciendo ese enfoque siempre en forma directa, ya sea hacia futuro o retroactivamente. Es la justificación jurídica del fenómeno "representación voluntaria". 
En relación con su naturaleza jurídica, en el ámbito de la representación voluntaria esta legitimación puede ser anterior o posterior al acto, señalando López de Zavalía (2000) que en el primer caso asume la forma de "autorización-poder", y en el segundo la de ratificación (autorización-ratificación).

Con la sanción del CCiv. y Com. puede sostenerse que la noción de ratificación y de poder, como situación jurídica que constituye o integra la legitimación de obrar resultan ser similares en cuanto a su naturaleza y oponibilidad a terceros. Así el artículo 369 señala que "(...) La ratificación suple el defecto de representación (...)", por lo cual estando el fenómeno representativo referido a una tercera parte con la cual se celebra el acto o hecho, la subsanación del defecto debe estar referida a ese tercero. Continúa el artículo afirmando que “(...) Luego de la ratificación, la actuación se da por autorizada, con efecto retroactivo al día del acto, pero es inoponible a terceros que hayan adquirido derechos con anterioridad (...)", por lo cual si resulta oponible a terceros que pretendiesen derechos con posterioridad.

El artículo 370 referido al tiempo de la ratificación, señala que

(...) la ratificación puede hacerse en cualquier tiempo, pero los interesados pueden requerirla, fijando un plazo para ello que no puede exceder de quince días; el silencio se debe interpretar como negativa. Si la ratificación depende de la autoridad administrativa o judicial, el término se extiende a tres meses. El tercero que no haya requerido la ratificación puede revocar su consentimiento sin esperar el vencimiento de estos términos (...).

Reconociéndose un interés jurídico a "los interesados", de requerirla, y hasta otorga la facultad de revocar su consentimiento a "los terceros que no haya requerido la ratificación", razón por la cual debe concluirse que la ratificación es también "recepticia". En consecuencia, pareciera que la diferencia no se encuentra allí, sino más bien en la producción de sus propios efectos jurídicos, ya que en la ratificación actúa otorgando eficacia con posterioridad al acto o hecho jurídico ratificado.

Betti (2000) por su parte señala que la diferencia radica en el momento en que se realizan en el proceso cronológico de celebración del acto jurídico: El poder es anterior al desarrollo de la conducta representativa refiriéndose a la legitimación del sujeto en el acto jurídico, mientras que la ratificación es posterior a la realización del acto, implicando una asunción por parte del interesado de las consecuencias jurídicas del acto celebrado sin poder. En este sentido, Mosset Iturraspe, señala que la ratificación es una “(...) autorización ex post facto que tiene el mismo efecto que la autorización previa (...)" (Mosset Iturraspe, 1998, p. 217), pudiéndose entender que participa de la tesitura que niega una diferencia sustancial. 
REPRESENTACIÓN, MANDATO Y PODER. UNA ENCRUCIJADA ENTRE EL DERECHO DE FORMA Y DE FONDo - MaURo F. Leturia, Solange C. Nugoli y Adrián E. GochicoA (Pp. 57-78)

https://doi.org/10.24215/25916386e003

Volviendo sobre la naturaleza jurídica, Lorenzetti (2003 y 2015) observa a la noción de poder como otorgante de legitimación para obrar en nombre ajeno. Betti, analizando los presupuestos del negocio jurídico, señala (entre otros) a la legitimación de la parte definiéndola como una “(...) competencia para alcanzar o soportar los efectos jurídicos de la reglamentación de intereses a que se ha aspirado, la cual resulta de una específica posición del sujeto respecto a los intereses que se trata de regular (...)" (Betti, 2000, p. 203).

A ello agrega que puede tratarse de una legitimación ordinaria o normal, cuando existe coincidencia entre el sujeto del acto jurídico y el titular de los intereses que se constituyen, modifican, transmiten o extinguen como consecuencia de aquel. O bien de una legitimación excepcional o anormal propia de situaciones donde no existe dicha coincidencia. Distingue dentro de esta legitimación excepcional, cuando es permitida por el ordenamiento jurídico en vista del legitimado como un derecho propio (por ejemplo los derechos del acreedor a subrogarse en los derechos del deudor) de cuando la misma es otorgada en interés del legitimante. Dentro de esta categoría se ubicaría el poder cuando no se permite, en virtud del interés del legitimante ni del legitimado sino del tercero con quien se contrató, la cual se da cuando el acto jurídico es otorgado por quien carece de legitimación pero el ordenamiento jurídico reconoce eficacia con el fin de tutelar los derechos de los terceros, siendo estos los casos de legitimación aparente.

En el ámbito de la representación voluntaria esta particular forma de legitimación para obrar por la cual se permite imponer un orden vinculante a determinados intereses ajenos se manifiesta como poder o el poder de representación.

A lo hasta aquí analizado vale agregar que la doctrina mayoritaria coincide en que el poder, como una forma particular de legitimación para obrar, constituye un acto jurídico unilateral o una declaración unilateral de voluntad destinada a terceros (recepticia) por la cual el poderdante autoriza a otra persona, apoderado, a actuar en nombre e interés de aquel, teniendo como efecto inmediato que los actos realizados por el apoderado recaigan en la órbita y patrimonio del poderdante. Diferenciándose de la representación como fenómeno jurídico, formando parte de este, en virtud del origen, su ámbito de aplicación, y la producción de sus efectos.

En cuanto a su origen, la representación puede ser legal, convencional o derivar de un estatuto, mientras que el poder tiene su fundamento en el principio de la autonomía de voluntad. En relación con el ámbito de aplicación la representación como fenómeno jurídico constituye un género que para poder plasmarse se encuentra asentado en la existencia de una particular forma de legitimación que tiene su origen en la ley, la voluntad o un estatuto, mientras que el poder como situación jurídica es la manifestación de esa particular forma de legitimación en el ámbito de la Representación voluntaria o convencional. 
Respecto a la producción de efectos jurídicos, en su concepción amplia la representación produce sus efectos de manera inmediata o mediata, mientras que el poder siempre de manera inmediata (esta diferencia puede verse entendiendo a la ratificación como una autorización ex post facto, la cual produce de manera inmediata los efectos mediatos de la representación).

Por su parte, Betti señala que presenta como características su independencia y abstracción, ya que resulta ajeno el otorgamiento del poder a “(...) la justificación económico-social que subyace en la relación de cooperación o de gestión (....)" (2000, p. 504), la cual puede derivar de diversos actos jurídicos (mandato, contrato de trabajo, entre otros). Mientras esta última se refiere a la relación interna entre el representado y el representante, el poder regula los efectos frente a los terceros. En suma, si bien existe una diferenciación conceptual entre la relación interna que existe entre representado y representante y la relación externa entre representado y tercero, puede suceder que ambas relaciones surjan de un mismo acto jurídico, de tal manera que puede surgir explícitamente como acto de apoderamiento o implícitamente en el acto jurídico que regula la relación interna entre el representante y representado.

Ahora bien, cierto sector de la doctrina entiende que el poder puede ser concebido no solo como un acto jurídico unilateral sino más bien como el instrumento en si o la forma de instrumentar las facultades. Por lo cual se entiende que el poder sería el instrumento que contiene la autorización que implica.

En relación con la forma, el acto de apoderamiento debe ser otorgado con las mismas formalidades que se requieren para la realización del acto jurídico a ejecutar. Así el artículo 363 del CCiv. y Com. señala que “(...) el apoderamiento debe ser otorgado en la forma prescripta para el acto que el representante debe realizar (...)". En este orden de ideas, el artículo 1017 del CCiv. y Com. señala una serie de actos que requieren escritura pública, por lo que el apoderamiento para realizar estos actos ha de realizarse en escritura pública.

\section{Mandato}

El mandato es un contrato, por lo tanto, un acto jurídico bilateral, mediante el cual dos o más partes manifiestan su consentimiento para crear, regular, modificar, transferir o extinguir relaciones jurídicas patrimoniales. Específicamente el artículo 1319 del CCiv. y Com. señala que “(...) Hay contrato de mandato cuando una parte se obliga a realizar uno o más actos jurídicos en interés de otra (...)”. Ello se completa contemplando situaciones fácticas que implican el otorgar o aceptar en forma tácita un contrato de mandato (Leturia, 2016). 

De FONdo - MaUro F. Leturia, Solange C. Nugoli y AdRián E. GochicoA (PP. 57-78) https://doi.org/10.24215/25916386e003

El CCiv. y Com. lo caracteriza como un contrato de cooperación o colaboración que según su finalidad socio-económica, puede ser oneroso o gratuito de conformidad a lo que pacten las partes pero si nada se ha pactado se presume oneroso (artículo 1322), no formal salvo que el acto jurídico cuya ejecución se encarga realizar tenga prevista una forma específica determinada por la ley (artículos 284, 285,1015 y 1017), consensual, ya que para su perfeccionamiento basta el acuerdo de las partes, bilateral, ya que implican obligaciones reciprocas para ambas partes (artículos 1324 y 1328).

En cuanto a la relación entre mandato y representación, en primer lugar, como se señaló dentro del fenómeno jurídico de la "representación", existen relaciones entre el representante y el representado, entre el representante y el tercero, y por último entre este tercero y el representado. Así, la representación se refiere exclusivamente a la relación entre el representante y el tercero, siendo ajenas a ellas la causa de la representación (poder, ratificación o autorización otorgada por la ley, ya sea como oficio o investidura), como las vinculaciones internas que se dan en ella entre representante y representado (por ejemplo contrato de mandato, contrato de trabajo, locación de servicios, entre otros). Por su parte, el mandato, siguiendo a Betti, se refiere al supuesto de “(...) justificación económico-social que subyace en la relación de cooperación o de gestión (...)” (2000, p. 504), la cual en la representación voluntaria puede derivar de diversos actos jurídicos y no solo del mandato (por ejemplo del contrato de trabajo, entre otros).

Señala Lorenzetti (2003) que la regulación legal del contrato se encuentra justificada en el resguardo de los bienes del mandante y en la limitación del actuar del mandatario. Mientras la representación pude tener su origen en la ley, la voluntad y un estatuto de una sociedad, el mandato encuentra su origen en la voluntad de las partes.

De la diferenciación conceptual existente entre representación, poder y mandato se deduce que puede existir mandato con representación o mandato sin representación, de acuerdo con que el mandato implique o no el otorgamiento de poder por parte de unas de las partes en forma implícita o expresa.

El artículo 1320 del CCiv. y Com. establece que si el mandante confiere poder para ser representado, deben aplicarse disposiciones previstas en el artículo 362 y siguientes referidas a la representación. Por otro lado, si no se otorga poder de representación, el mandatario actúa en nombre propio pero en interés ajeno, aplicándose las disposiciones citadas a la relación entre mandante y mandatario siempre que no resulte modificadas por las disposiciones referidas al contrato de mandato. 
El artículo 1321 del CCiv. y Com. establece que cuando el mandato se carece de representación, el mandatario actúa en nombre propio pero en interés del mandante. Este último no queda obligado directamente respecto del tercero, ni este respecto del mandante. El mandante puede subrogarse en las acciones que tiene el mandatario contra el tercero, e igualmente el tercero en las acciones que pueda ejercer el mandatario contra el mandante. Por lo cual, no surgen obligaciones entre mandante y terceros, pero sí se prevé la existencia acciones de subrogación.

En cuanto a la relación entre mandato y poder, como se señaló, el poder es un acto jurídico unilateral o una declaración unilateral de voluntad, mientras que el mandato es un contrato, esto es un acto jurídico bilateral. Lorenzetti señala que “(...) las relaciones entre el poder y el negocio base que le da origen es de conexidad, porque comparten su causa (...)" (2003, p. 182). Siguiendo esta idea, no siempre existe una tajante separación entre el negocio base que regula la relación interna y el acto de concesión de poderes. Pueden hallarse fundidas ambas declaraciones de voluntad, como ejemplo un mandato destinado a exhibirse a terceros que lleva implícito el otorgamiento del poder.

\section{Forma del poder, del mandato y la regulación procesal}

\section{V.1. Sobre la forma del poder y el mandato}

$\mathrm{Al}$ respecto, la regla se asienta en el principio de libertad de formas establecida en el artículo 284 del CCiv. y Com., reiterada para los contratos en el artículo 1015. Sobre esta base puede distinguirse entre actos jurídicos (y contratos) no formales y actos jurídicos (y contratos) formales. Dentro de estos últimos, se puede diferenciar según la forma sea exigida para la prueba, o para la validez del acto ya sea en forma relativa o absoluta.

Específicamente el CCiv. y Com. respecto a la forma del poder, en el artículo 363 del CCiv. y Com. señala que “(...) el apoderamiento debe ser otorgado en la forma prescripta para el acto que el representante debe realizar(...)". Por lo cual, el principio general es que el poder se trata de un acto jurídico no formal, debiendo quedar únicamente sujeto a formalidades cuando la ley lo disponga para el acto que el representante deba realizar.

En este orden de ideas, por ejemplo el artículo 1017 del CCiv. y Com. señala una serie de actos que requieren escritura pública, por lo que el apoderamiento para realizar estos actos debe realizarse en escritura pública, entre ellos el poder para contratos que tienen por objeto la adquisición, modificación o extinción de derechos reales sobre inmuebles; a diferencia del Código de Vélez, que en el artículo 1184 inc. 7 referido a la forma de los contratos establecía la formalidad de 
https://doi.org/10.24215/25916386e003

la escritura pública para “(...) Los poderes generales o especiales que deban presentarse en juicio, y los poderes para administrar bienes, y cualesquiera otros que tengan por objeto un acto redactado o que deba redactarse en escritura pública (...)", no resulta necesario que los poderes para presentarse en juicio sean otorgados en escritura pública.

Con relación a la forma en el contrato de Mandato, el CCiv. y Com. no contiene una norma expresa y específica para este contrato. Por lo cual deben aplicarse, por un lado, la regla general para los actos jurídicos y para los contratos en cuanto a la forma. Así partiendo del principio de libertad de formas (artículos 284 y 1015), solo será formal cuando el contrato de mandato sea con representación por remisión del artículo 1320 del CCiv. y Com. Esto en virtud de resultar aplicable la regulación sobre representación artículo 362) siendo en este caso un contrato formal, ya que rige el principio de accesoriedad de la forma (artículo 363 y 1017 inc. C), por lo que debe realizarse en escritura pública por ser accesorio de otros contratos cuando estos sean otorgados en escritura pública.

En síntesis, cuando el apoderamiento se otorgue sin un negocio base de mandato rige la regla de la libertad de formas, y como excepción resulta ser un acto formal en virtud del principio de accesoriedad de forma. En cuanto al mandato, la regla es que se trata de un contrato no formal rigiendo la regla de libertad de formas y excepcionalmente será un contrato formal exigiéndose que sea otorgado por escritura pública.

\section{V.2. La regulación procesal de la representación}

Estando vigente el código de Vélez, las respectivas jurisdicciones provinciales y la nacional dictaron sus códigos procesales en lo civil y comercial, siguiendo al derecho de fondo regulado.

Ejemplo de ello son los Códigos Procesales Civil y Comercial de la provincia de Buenos Aires y el de Nación, donde se señala la necesidad de acreditar la representación que se invoca cuando se intervenga en el proceso por un derecho que no es propio. De este modo, el artículo 46 alude a "acompañar los documentos que acrediten el carácter que enviste", y el artículo 47 señala que

(...) Los procuradores o apoderados acreditarán su personalidad desde la primera gestión que hagan en nombre de sus poderdantes, con la pertinente escritura de poder. Sin embargo, cuando se invoque un poder general o especial para varios actos, se lo acreditará con la agregación de una copia íntegra firmada por el letrado patrocinante o por el apoderado. De oficio o a petición de parte, podrá intimarse la presentación del testimonio original (...). 
A diferencia del actual CCiv. y Com. el Código de Vélez no desarrolló una teoría general de la representación, pero si exigió la escritura pública como forma para otorgar poder para estar en juicio (artículo 1184 inc. 7).

Durante la vigencia del Código de Vélez, la doctrina imperante entendió que los artículos 46 y 47 de los códigos de rito se referían a que la acreditación (de la representación procesal) era mediante la presentación de escritura pública y no con una copia simple salvo para la excepción contemplada (cuando se invoque un poder general o especial para varios actos, caso en el que se podía acreditar con la agregación de una copia íntegra firmada por el letrado patrocinante o por el apoderado). Dicha exigencia, surgía de la interpretación conjunta que se hacía de los artículos 46 y 47 de los Códigos de Procedimiento Civil y Comercial y el derogado 1184 inc. 7 del Código de Vélez.

El reciente CCiv. y Com. no determina exigencia alguna respecto a que los poderes para estar en juicio deban tener como forma la escritura pública, asimismo, se insiste en que para el contrato de Mandato, el CCiv. y Com. no contiene una norma expresa que permita calificarlo como un contrato formal. Por lo cual debe aplicarse la regla general - libertad de las formas - para los actos jurídicos y los contratos.

Podría plantear dudas la exigencia de la forma de escritura pública para actos que sean accesorios de otros contratos o actos otorgados en escritura pública y para cualquier contrato o acto jurídico que así deba otorgarse, por acuerdo de partes o disposición de la ley. En rigor de verdad, ningunos de esos casos abarca al mandato con poder de representación para estar en juicio o al poder judicial (otorgado sin un negocio base de mandato), en tanto ninguno de los citados códigos procesales exige como forma de los actos jurídicos procesales, sean otorgados en escritura pública, por lo que la regla de accesoriedad no resulta aplicable. En cuanto al segundo supuesto, esto es una exigencia de la ley, podría entenderse erróneamente que el nuevo código posibilitó que cada jurisdicción pueda en sus códigos procesales (leyes) exigir que sean otorgado en escritura pública.

Este razonamiento implica sostener, contra el texto expreso de la ley, que los artículos 46 y 47 son los supuestos en que la ley exige la escritura pública para los poderes judiciales. Lo cual no es correcto, ya que se habla de "documentos que acrediten" (artículo 46) y de la "la pertinente escritura de poder". El artículo 1017 del CCiv. y Com. en cuanto menciona que cualquier ley exija el otorgamiento del contrato o de un acto sea en escritura pública lo hace en clara referencia a que dicha exigencia se refiere a la forma, no a la posibilidad de acreditar en juicio el acto de la representación, ya que esta materia es propia de cada jurisdicción. 
En segundo lugar, es sabido que el documento es un género del cual participan, cuando tienen la forma escrituraria los instrumentos públicos y los instrumentos particulares, dentro de estos los instrumentos privados cuando son firmados por las partes (artículos 286 y 287 del CCiv. y Com.). Dentro de los instrumentos públicos se encuentra la escritura pública, la cual es un instrumento público que para considerarse escritura pública debe cumplir con ciertos requisitos (artículo 289, 290, 299 a 312 y concordantes del CCiv. y Com.).

Del texto de los artículos puede inferirse que a efectos de acreditar en juicio la personería que se invoca cuando se va a actuar procesalmente en nombre de otro la exigencia de que el poder conste en escritura pública no era un requisito esencial. Nótese que el artículo 47 señala como excepción que

Sin embargo, cuando se invoque un poder general o especial para varios actos, se lo acreditará con la agregación de una copia íntegra firmada por el letrado patrocinante o por el apoderado. De oficio o a petición de parte, podrá intimarse la presentación del testimonio original (...).

Por lo que, la posibilidad de que se intime a presentar el "testimonio" pone de manifiesto, por un lado, que el artículo 47 se refiere a la acreditación en juicio de la representación invocada la cual puede hacerse hasta con una copia firmada y no a la forma como elemento del acto jurídico del que surge la representación procesal. Y, por el otro, que la exigencia de la escritura pública para poderes judiciales surgía exclusivamente del artículo 1184 inc. 7, y no de los artículos 46 y 47 de los respectivos Códigos Procesales.

Por otro lado en la provincia de Buenos Aires se ha instaurado el Sistema de Notificaciones y Presentaciones Electrónicas de la Suprema Corte de la provincia de Buenos Aires, por el cual los abogados apoderados, mediante poder general o poder especial, pueden enviar presentaciones electrónicas a los organismos jurisdiccionales, ya sean actos procesales de mero trámite o actos procesales impulsorios o peticiones, bastando para ello que se encuentre acreditado en el expediente la calidad de apoderado.

Por la resolución de la S.C.B.A. 3415/12 se aprobó el protocolo 1827/12 que establece el "Reglamento para las presentaciones electrónicas", aprobándose el actapoder que se debe suscribir (las partes del proceso) para facultar a los abogados a realizar las presentaciones electrónicas de carácter impulsorio o que impliquen una petición de la parte. Expresamente en el punto 2 referido a la adhesión, trámites y efectos se establece que 
A los fines de que los peticionantes que actúen por derecho propio confieran poder suficiente a su letrado para realizar presentaciones electrónicas y no se encuentren comprendidos en los supuestos enunciados por los artículos 46 y 85 del CPCC, la parte deberá, en el momento de producirse la adhesión conferir poder suficiente frente al Secretario labrándose el acta pertinente. Este mecanismo se justifica en la condición ad probationem o relativa que posee la formalidad prescripta por el artículo 1184 inc. 7 del Código Civil.

Por lo cual durante la vigencia del Código de Vélez, hasta agosto del 2015, bajo este sistema, hoy obligatorio, el poder para presentaciones electrónicas se podía otorgar por acta ante el Secretario del Juzgado, o sea en un instrumento público (artículo 979 del Código de Vélez, hoy 289 del CCiv. y Com.) y no por escritura pública. Como se indicó se derogó el artículo 1184 inc. 7, y su reemplazo en el CCiv. y Com. el artículo 1015 no contempla como forma de los poderes generales o especiales para juicios que sean otorgados en escritura pública. Por lo cual, con la vigencia del nuevo código ya no resultaría necesaria ni el acta por ante el secretario para otorgar poder para presentaciones electrónicas que excedan las de mero trámite.

\section{Jurisprudencia}

A continuación se realiza una breve referencia a la jurisprudencia sobre la subsistencia o no de la exigencia de que los poderes generales o especiales sean otorgados en escritura pública.

\section{VI.1. Fallos a favor de la continuidad de la exigencia u obligatoriedad del otorgamiento de poderes generales o especiales mediante escritura pública}

- CACC., San Isidro, sala III, 25/02/2016, "OROPEL, CLARA ANGÉLICA C/ GÓMEZ, RAÚL ALBERTO S/ ACCIÓN DECLARATIVA”. SI-39362-2014.

- C.Civ., sala H, 03/05/2016, "ARROYO, NICOLAS SEBASTIÁN c/ DREID, CARLOS ARTURO Y OTRO s/PRUEBA ANTICIPADA". Exp. 33630/2015.

- CACC., Mar del Plata, sala II, 31/05/2016, "GRIPPALDI ALFREDO ANTONIO C/ CONS. PROP. EDIFICIO SANTA LUCIA S/ COBRO DE SUMARIO DE SUMAS DE DINERO". Exp. 161144.

- C.Civ., sala M, 27/03/ 2017, "BARREYRO, MARÍA LAURA c/ PULICE, DIEGO SEBASTIÁN Y OTROS s/DAÑOS Y PERJUICIOS - RESP. PROF. MÉDICOS Y AUX.” Exp. 64880/2015. 
— CACC., sala I, Azul, 18/05/ 2017, "GONZÁLEZ HUGO ALBERTO C/ CASTELLANO YANEL ANAHÍ Y OTRO S/ DAÑOS Y PERJ. INCUMP. CONTRACTUAL (EXC. ESTADO)". Exp. №: 6-2169-2017.

— CACC., sala II, Mar del Plata 13/07/2017, "MIRANDA MIGUEL RAMÓN C/ VISVEQUI JUAN S/COBRO EJECUTIVO”. Exp. 163673.

\section{VI.2. Fallos en contra de la obligatoriedad del poder general o especial otorgado en escritura pública}

—CACC., Dolores, 04/02/2016, “FOCKE, TEÓFILO S/ SUCESIÓN”. Exp. 94.923.

- CACC., Dolores, 11/02/2016, "GIGENA, SILVIA GLADYS C/ GIGENA, PERLA NANCY S/ DIVISIÓN DE CONDOMINIO”. Exp. 95.004.

- CACC., La Plata, 10/03/2016, "ORTIZ ROBERTO Y OTROS C/ SASSAROLI ANA MARÍA Y OTRO/A S/PROPIEDAD HORIZONTAL - CUESTIONES E/PROPIETARIOS. REG. INTER. No 40 /16, LIBRO INTERLOCUTORIOS LXXII. JDO. 14". Exp. No 119961.

- CACC., II, Sala Segunda, La Plata, (16/06/2016) "SCIATORE DIEGO MARTIN Y OTRO/A C/ ROSSINI ESTELA LAURA Y OTRO/A S/ DAÑOS Y PERJ. AUTOM. C/ LES. O MUERTE (EXC. ESTADO)". Exp. 120.272.

- CACC., sala I, San Isidro, 06/12/2016 “G. G. M. c/ F. M. E. s/ Petición de herencia" No SI-9392-2010 (J.8).

- SCBA., 08/02/2017, "CARNEVALE COSME OMAR C/ PROVINCIA DE BUENOS AIRES S/ PRETENSIÓN INDEMNIZATORIA - RECURSO EXTRAORDINARIO DE INAPL. DE LEY".

\section{El Anteproyecto de Código Procesal Civil, Comercial y de Familia de la provincia de Buenos Aires}

En la provincia de Buenos Aires el Anteproyecto del Código Procesal Civil, Comercial y de Familia de la provincia de Buenos Aires fue redactado por el Colegio de Abogados de La Plata y firmado por los Dres. Francisco Agustín Hankovits, Juan Manuel Hitters, Pablo Agustín Grillo Ciocchini, Mariela Panigadi, y Andrés Antonio Soto, refiriéndose al tema en cuestión en el Libro I, Título 2, Capítulo 2 referido a la Representación Procesal en su artículo 43 señala que a efectos de justificar la personería invocada: 
(...) La persona que se presente en juicio por un derecho que no sea propio, aunque le competa ejercerlo en virtud de una representación legal, deberá acompañar con su primer escrito los documentos que acrediten el carácter que inviste. Sin embargo, los padres que comparezcan en representación de sus hijos, no tendrán obligación de presentar las partidas correspondientes, salvo que el juez, a petición de parte o de oficio los emplazare a presentarlas, bajo apercibimiento del pago de las costas y perjuicio que ocasionaren. La representación en juicio podrá instrumentarse mediante poder notarial o acta labrada ante el funcionario que al efecto designe el Colegio de Abogados de cada Departamento judicial, con la comparecencia del poderdante y el profesional que actuará como apoderado y previa verificación de la matrícula de este último. Cuando el valor pecuniario de los juicios no supere un valor equivalente de ciento veinte (120) Jus, el trámite será gratuito.

Y su artículo 44 indica que

(...) Los procuradores o apoderados acreditarán su personalidad desde la primera gestión que hagan en nombre de sus poderdantes, con la pertinente escritura de poder o con el acta labrada ante el funcionario que a tal efecto designe el Colegio de Abogados de cada Departamento Judicial.

Sin embargo, cuando se invoque un poder general o especial para varios actos, se lo acreditará con la agregación de una copia íntegra firmada por el letrado patrocinante o por el apoderado. De oficio o a petición de parte, podrá intimarse la presentación del testimonio original. No será necesario el carácter de apoderado para la realización de actos de mero trámite. Todas las presentaciones judiciales serán consideradas de mero trámite con excepción de (I) la demanda, contestación, reconvención y su contestación; (II) el allanamiento, desistimiento, transacción o conciliación; (III) la interposición de recursos extraordinarios.

Como puede apreciarse, en el anteproyecto se opta por otra opción, cuanto menos curiosa: el otorgamiento del poder en acta labrada ante el funcionario que al efecto designe el Colegio de Abogados de cada Departamento judicial, con la comparecencia del poderdante y el profesional que actuará como apoderado y previa verificación de la matrícula de este último. Curioso por cuanto termina constituyendo una formalidad no exigida por el CCiv. y Com., y más curioso aún es que esta "formalidad" o "trámite" es oneroso: solo será gratuito cuando el valor pecuniario de los juicios no supere un valor equivalente de ciento veinte (120) Jus. 
REPRESENTACIÓN, MANDATO Y PODER. UNA ENCRUCIJADA ENTRE EL DERECHO DE FORMA Y De Fondo - Mauro F. Leturia, Solange C. Nugoli y Adrián E. GochicoA (pP. 57-78) https://doi.org/10.24215/25916386e003

¿Deja de ser negocio exclusivo de los Notarios para ser negocio de los Colegios de Abogados?, cuando el nuevo Código no exige formalidad alguna.

\section{Bases para la Reforma Procesal Civil y Comercial para el "Anteproyecto de Nuevo Código Procesal Civil y Comercial de la Nación”}

Por su parte, a nivel nacional por resolución 829-E/2017 el Ministro de Justicia Garavano en el marco del expediente No EX -2017-15990702-APN-MJ del registro de ese Ministerio (y de conformidad a las Resoluciones M. J. y D. H. No 151 del 30 de marzo de 2016, 441 del 24 de junio de 2016, RESOL-2016-546 -E- APNMJ del 28 de julio de 2016 y RESOL-2017-496 - APN-MJ del 3 de julio de 2017) aprueba "las Bases para la Reforma Procesal Civil y Comercial", el cual constituye un documento elaborado por la Comisión Redactora del Nuevo Código Procesal Civil y Comercial de la Nación, donde se delinean las ideas madres que servirán para la elaboración de un "Anteproyecto de Nuevo Código Procesal Civil y Comercial de la Nación".

En dichas bases para la Reforma Procesal Civil y Comercial en el capítulo VII referido a la actividad procesal y a los actos de postulación, en el punto 3 referido a las Partes postula los siguientes lineamientos:

a) Patrocinio letrado: Es necesario asegurar el asesoramiento profesional para las partes, como mejor modo de lograr el acceso a la justicia y la garantía de la defensa en juicio. Sobre esa base, es necesario establecer el modo de la participación del abogado en el proceso; sea como patrocinante, como apoderado, o como defensor de la parte con facultades para efectuar peticiones en su nombre. b) Acreditación de la representación para actuar en juicio Se mantendrá la doble forma de actuación vigente en el actual CPCC: (I) de la parte, por su propio derecho, con patrocinio obligatorio, y (II) del abogado con poder para actuar en juicio en representación de la parte. La actuación por derecho propio, o la actuación del apoderado, es necesaria sólo para ciertos actos puntuales y que implican la disposición de derechos (demanda, contestación, interposición o contestación de incidentes, desistimiento, transacción, conciliación y demás actos de trascendencia semejante).

En cambio, para todos los demás actos del proceso, basta la designación como abogado de la parte. Así el abogado, con esta sola designación, puede ejercer todos los actos procesales en nombre de su mandante, con excepción de los específicamente mencionados. En cuanto a la forma del mandato, en virtud de la modificación establecida por el Código Civil y Comercial, no es necesario, ya que el poder para actuar en juicio sea extendido en escritura pública. Pero sí debe constar por escrito. 
Puede apreciarse que si bien es una idea o base, es conteste con la no exigencia de la formalidad de que los poderes judiciales deban constar en escritura pública, siendo contrario no solo al proyecto de reforma de la Provincia de Buenos Aires que crea otra "formalidad", sino a la corriente jurisprudencial en el ámbito de la Justicia Nacional.

\section{Conclusión}

En un primer análisis, la temática plantea una cuestión sobre la constitucionalidad de que los Códigos Procesales puedan o no exigir "formalidades" para los actos jurídicos regulados por el CCiv. y Com. Así las cosas, por un lado, el nuevo Código incorpora normas procesales conjuntamente con las normas de sustanciales. Por el otro, como en la temática del presente artículo, las normas sustanciales ceden un espacio antes regulados por ellas, la cual en principio parecería ser materia propia de las normas procesales.

En el supuesto de los poderes judiciales, ya la cuestión no se plantea por una "invasión" de un Código de fondo en un ámbito no delegado por las Provincias, sino en un retroceso o cese de ese espacio antes ocupado, por lo que se plantea si puede cada Jurisdicción Provincial "invadirlo" o "recobrarlo". No escapa el entendimiento que el nuevo Código Civil y Comercial también regula la misma materia que su antecesor derogado, pero esta vez por "omisión": Resultan menos exigente en cuanto a ciertos elementos de los actos jurídicos, género al que no escapan los actos procesales.

Queda claro que ya no debe requerirse la escritura pública para el otorgamiento de los poderes que preveía el artículo 1184 inc. 7 del Código de Vélez (artícu$\operatorname{los} 7,363,1015,1017$, CCiv. y Com.). Pero frente a esto, por un lado, se sostiene que si bien actualmente el nuevo Código Civil y Comercial de la Nación no incluye al mandato para los poderes generales o especiales para estar en juicio entre los actos a instrumentarse por escritura pública (artículos 1015 y 1017 CCiv. y Com.), no importa por sí la derogación expresa o tácita de las normas que emergen de los Código Procesales (artículos 75 inc. 12, 121 de la Constitución Nacional). Por otro lado, se puede sostener lo contrario: el Código Civil y Comercial de la Nación consagra el principio de libertad de formas al respecto (artículos 284, 285, 363, 1319 del CCiv. y Com.) y es a través del análisis específico de cada acto jurídico el que determinará qué forma debe revestir el acto de apoderamiento.

Las provincias han delegado la facultad de dictar el Código Civil y Comercial al Congreso de la Nación y teniendo en cuenta el carácter netamente procesal de las reglas que sobre la acreditación del mandato establece el artículo 47 del Código Procesal Civil y Comercial de la provincia de Buenos Aires y el de Nación 

De FONdo - MaUro F. Leturia, Solange C. Nugoli y AdRián E. GochicoA (PP. 57-78) https://doi.org/10.24215/25916386e003

(que fue redactado en consonancia con el articulado del anterior Código Civil, artículo 1184 inciso 7), no resulta admisible que la legislación local limite el alcance establecido por la normativa de fondo (conf. artículos 31 y 75 inc. 12 de la Constitución Nacional; cfme. Ac. 79.617 del 18/4/2001). Al haberse sancionado un nuevo Código Civil y Comercial de la Nación por parte del Congreso Nacional en ejercicio de las facultades delegadas (ley № 26.994), en el que no se exige expresamente el instrumento público para la acreditación del mandato para intervenir en juicio (artículos 1015 y 1017 del CCiv. y Com.), a su disposición ha de estarse. Así las cosas, una ley procesal que no puede crear para actos jurídicos -en la especie: contrato de mandato-, formas instrumentales que la ley sustancial no prevé (artículos 5, 31, 75 inc. 12, 121, 126, Constitución Nacional). Es decir, las Jurisdicciones provincial y la nacional no puede imponer las formas a los contratos, cuando ellas no están previstas en la ley nacional que regula sobre la materia delegada.

La doctrina constitucionalista en general entiende que la función de legislar sobre materia procesal pertenece, en principio, a cada una de las Provincias y no al Congreso de la Nación. Conforme al artículo 75, inciso 12 de la Constitución Nacional, el derecho común está formado por los códigos y las leyes dictadas por el Congreso Nacional pero no deben alterar las jurisdicciones locales. Por su parte, el artículo 121 de la Constitución Nacional señala que las Provincias conservan todo el poder no delegado al Gobierno Nacional. Frente a esto la Corte Suprema de Justicia de la Nación ha señalado que esa atribución de legislar el derecho común que las Provincias han delegado a la Nación legitima la regulación (por acción y por omisión) de aspectos procesales en determinadas situaciones (CSJN, Fallos 138:157; 141:254; 162:376; 247:524; 265:30, entre otros), cuando lo considere el caso, y así puede prescribir formalidades especiales para el ejercicio de determinados derechos establecidos en los códigos fundamentales que le incumbe dictar. ("Bernabé Correa" Fallos 138, p. 157).

\section{Bibliografía}

Alterini, A. A. (1999). Contratos civiles - comerciales - de consumo. Teoría general. Argentina: Abeledo-Perrot.

Betti, E. (2000). Teoría General del Negocio Jurídico. Argentina: Editorial Comares.

Borda, A. (2016). Derecho civil. Contratos. Argentina: La Ley.

Camps, C. E. (2004). Código Procesal Civil y Comercial de la Provincia de Buenos Aires -anotado - comentado - concordado. Argentina: LexisNexis - Depalma.

Centanaro, E. (2015). Manual de Contratos. Argentina: La Ley. 
Falcón, E. M. (2016). Capacidad, representación y legitimación. La representación procesal en el Código Civil y Comercial. Revista de Derecho Procesal. Argentina: Rubinzal-Culzoni.

Ghersi, C. A. (1998). Contratos Civiles y Comerciales. Argentina: Astrea.

Herrera, M.; Caramelo, G. y Picasso, S. (2015). Código Civil y Comercial de la Nación Comentado. Argentina: Infojus.

Leturia, M. F. y Jalil, S. (2016). Manual del Martillero y del Corredor. Argentina: Librería Editora Platense.

López De Zavalía, F. J. (1997). Teoría de los contratos. T. I. Parte General. 4a edición. Argentina: Zavalía.

Lorenzetti, R. L. (2003). Contratos. Parte especial. Argentina: Rubinzal-Culzoni.

Lorenzetti, R. L. (2015). Código Civil y Comercial de la Nación Comentado. Argentina: Editorial Rubinzal-Culzoni.

Mosset Iturraspe (1998). Contratos. Buenos Aires: Rubinzal-Culzoni Editores.

Rivera y Crovi (2016). Derecho Civil, Parte general. Buenos Aires: Abeledo Perrot.

Rivero, J. C. y Medina, G. (2015). Código Civil y Comercial Comentado. Buenos Aires: La Ley.

Fecha de recepción: 06-02-2019

Fecha de aceptación: 18-08-2019 